\title{
Magnetoelastic Anisotropy in Glass-Coated Microwires Studied using SAMR Method
}

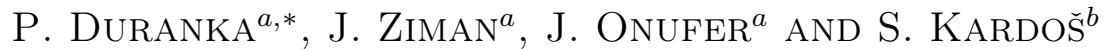 \\ ${ }^{a}$ Department of Physics, Faculty of Electrical Engineering and Informatics, Technical University of Košice, \\ Park Komenského 2, 04200 Košice, Slovakia \\ ${ }^{b}$ Department of Technologies in Electronics, Faculty of Electrical Engineering and Informatics, \\ Technical University of Košice, Park Komenského 2, 04200 Košice, Slovakia
}

\begin{abstract}
A small-angle magnetization rotation (SAMR) experiment carried out on a single piece of glass-coated microwire is presented. The specific conditions of this experiment are discussed. The cylindrical shape of the microwire with small cross-sectional area means that a transverse AC magnetic field of relatively high frequency created by an electric current flowing through the microwire has to be used. It is shown that this experiment provides an opportunity to determine magnetoelastic anisotropy and corresponding axial mechanical stress in the metallic core of the wire. The difference in thermal expansion coefficients between the glass coating and the metallic core causes the magnetoelastic anisotropy field to increase with decreasing temperature. This behaviour, as well as the effect of stress relaxation by annealing, are demonstrated in this experiment based on the SAMR method.
\end{abstract}

DOI: 10.12693/APhysPolA.137.868

PACS/topics: microwire, SAMR method, magnetoelastic anisotropy

\section{Introduction}

Cylindrical ferromagnetic amorphous wires form a group of modern magnetic materials which due to their unique magnetic properties [1-4] attract attention for potential sensor applications $[5,6]$. Glass-coated microwires are a special sub-group of these materials. They are prepared using the Taylor-Ulitovski technique [7]. In order to obtain an amorphous state in their metal core, both glass coating and metal core are rapidly cooled in the process of preparation. The difference in thermal expansion coefficients of the glass coating and metallic core results in strong tensile mechanical stress $[2,8]$ in the metal core in the as-quenched state [3]. Tensile stress combined with positive magnetostriction means that strong magnetoelastic anisotropy with axial easy axis is induced. The microwires with positive magnetostriction typically have a rectangular hysteresis loop, and they exhibit so-called bi-stable behaviour. Magnetization reversal starts with the depinning of a single domain wall from the closure domain structure at the microwire ends [1-4, 8]. These microwires also provide an opportunity to study the dynamics of a single domain wall between axial domains propagating along the microwire. All these processes can be strongly influenced by the magnitude of magnetoelastic anisotropy. Knowledge of the field of this anisotropy could provide useful parameters for interpretation of magnetization reversal in this type of microwire.

The small-angle magnetization rotation (SAMR) method is usually used to measure the saturation magnetostriction in amorphous ferromagnetic ribbons [9].

*corresponding author; e-mail: peter.duranka@tuke.sk
From the changes in the field of magnetoelasic anisotropy caused by the applied tensile stress the saturation magnetostriction is determined. As already mentioned above the conditions in this experiment are very similar to the situation in a glass-coated microwire prepared by rapid quenching from the melt $[10,11]$. In this paper the results obtained using the SAMR method for the study of glass-coated microwire are reported.

\section{Experimental}

In the original experiment [9] the experimental set-up consisted of three coils, two magnetizing coils, and one pick-up coil. One magnetizing coil is coaxial with the pick-up coil, and its role is to saturate the sample magnetically (ribbon in the original experiment) by means of a DC field. AC current of small amplitude flows through the second coil, and it creates an $\mathrm{AC}$ field perpendicular to the DC one (in the ribbon plane in the original experiment). If $f$ is the frequency of the AC field, then voltage with frequency $2 f$ is induced in the pick-up coil. Using the calculations presented in [9], it is possible to express this voltage as

$$
\varepsilon_{2 f}=\frac{1}{2} z S \mu_{0} M_{s} \omega A_{1}^{2} \sin (2 \omega t),
$$

where $\omega=2 \pi f, z$ is the number of turns in the pick-up coil, $S$ is sample cross-section area, $M_{s}$ is the saturation magnetization, and

$$
A_{1}=\frac{H_{\mathrm{AC} 0}}{\left(H_{\mathrm{DC}}+H_{\sigma}+H_{s}\right)},
$$

where $H_{\mathrm{AC} 0}$ is the amplitude of the perpendicular $\mathrm{AC}$ field, $H_{\mathrm{DC}}$ is the applied DC field, and $H_{s}$ is the field of shape anisotropy. Finally $H_{\sigma}=\frac{3 \lambda_{s} \sigma}{\mu_{0} M_{s}}$ is the field of magnetoelastic anisotropy, where $\sigma$ is tensile stresss, and $\lambda_{s}$ is saturation magnetostriction. 
In comparison with experiments performed on ribbons, there are some important differences which have to be taken into account when considering a similar experiment using cylindrical microwire. The radius of microwires is typically small (about $10 \mu \mathrm{m}$ or even less), which means that the cross-sectional area of the microwire, and as well as the amplitude of the measured signal are correspondingly small. The situation can be improved if an AC field of higher frequency is used. On the other hand, the shape anisotropy (transverse demagnetizing factor) is high for cylindrical samples so it is difficult to create an AC field with high enough amplitude using an external coil. It is even more difficult if a high-frequency field is needed. This problem can be solved if, instead of external coils, the AC field is created by alternating current flowing through the microwire itself [11]. In this case, the field of shape anisotropy in (2) can be neglected.

As mentioned above, the sensitivity of the experiment can be improved if a higher-frequency AC field is used. The limit for increasing the AC field frequency can be deduced from the relation between the microwire radius and skin effect depth. A rough estimation of skin effect depth $\delta$ can be made if we substitute the expression for circular susceptibility [12]

$$
\chi_{\varphi}=\frac{M_{s}}{H_{\mathrm{DC}}+H_{\sigma}}
$$

into formula

$$
\delta=\sqrt{\frac{1}{2 \pi f} \frac{2 \rho}{\mu_{0}\left(1+\chi_{\varphi}\right)}},
$$

where $\rho$ is wire resistivity, and $\mu_{0}$ is permeability of free space.

For the parameters of the microwire used in our experiment, i.e., $\mu_{0} M_{s}=1.55 \mathrm{~T}, \rho=1.3 \times 10^{-6} \Omega \mathrm{m}$, the ratio of skin depth over wire radius for five frequencies is shown in Fig.1. As can be seen, for axial fields higher than $2 \mathrm{kA} / \mathrm{m}$ the skin depth at all frequencies is more than three times larger than the wire radius.

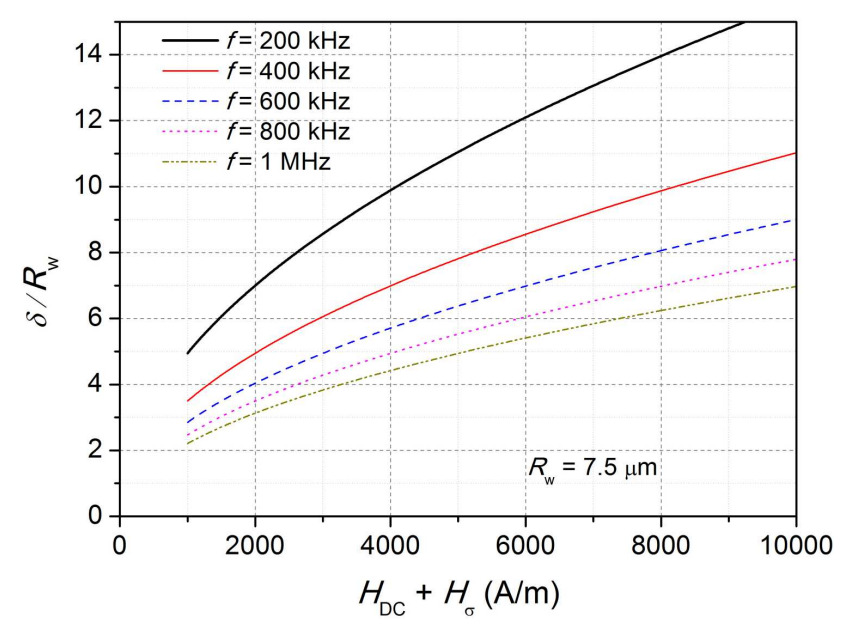

Fig. 1. Ratio of skin depth over wire radius $R_{w}$ as a function of axial field for five frequencies.

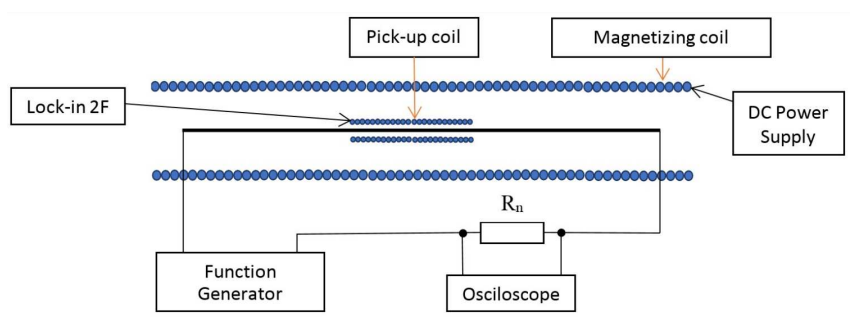

Fig. 2. Experimental set-up.

The experimental set-up used in our experiment is shown in Fig. 2. It consists of a magnetizing coil and a thin (diameter $0.5 \mathrm{~mm}$ ), long $(5 \mathrm{~cm})$ pick-up coil with 1840 turns. Thin copper leads were attached to the microwire ends with silver paint so that, using a function generator, $\mathrm{AC}$ current with amplitude of a few $\mathrm{mA}$ could flow through the microwire. Voltage with frequency $2 f$ induced in the pick-up coil was measured using a lockin amplifier. For measurements at temperatures below room temperature the set-up described in $[3,8]$ was used. A small modification of this set-up allowed measurements at temperatures slightly above room temperature to be taken.

A glass coated $\mathrm{Fe}_{77.5} \mathrm{Si}_{15} \mathrm{~B}_{7.5}$ microwire with total diameter of $30 \mu \mathrm{m}$ and metal core with diameter of $15 \mu \mathrm{m}$ was used in our experiment. We present the results of measurements on an as-quenched sample and on a sample annealed twice at the temperature of $350{ }^{\circ} \mathrm{C}$. The first annealing lasted two hours, and the sample was cooled slowly (approximately two degrees per minute) to room temperature. The second annealing lasted one hour, and then the sample was removed from the furnace, so the cooling to room temperature was much faster.

\section{Results and discussion}

In the SAMR experiment, the frequency of induced voltage $\varepsilon_{2 f}$ in the pick-up coils is two times higher than the transverse AC field frequency, and it is measured as a function of the applied axial field. A summary of this type of experimental dependence measured on the same piece of microwire is shown in Fig. 3. If the basic conditions of the experiment (axial saturation of the sample, small angle of magnetization rotation) are fulfilled, the dependence of the inverse value of the square root of the amplitude of induced voltage $\varepsilon_{2 f}$ should be a linear function of the applied axial field (see (1)). This type of experimental dependence is summarized in Fig. 4, and in fact, the linearity of these dependencies is very good. As mentioned above, the field of shape anisotropy (see (2)) can be neglected in our experiment. From the parameters of the linear dependencies fitted to the experimental data in Fig. 4 the magnitude of anisotropy fields $H_{\sigma}$ can be determined. For glass-coated amorphous microwires with positive magnetostriction it is predominantly a magnetoelastic 


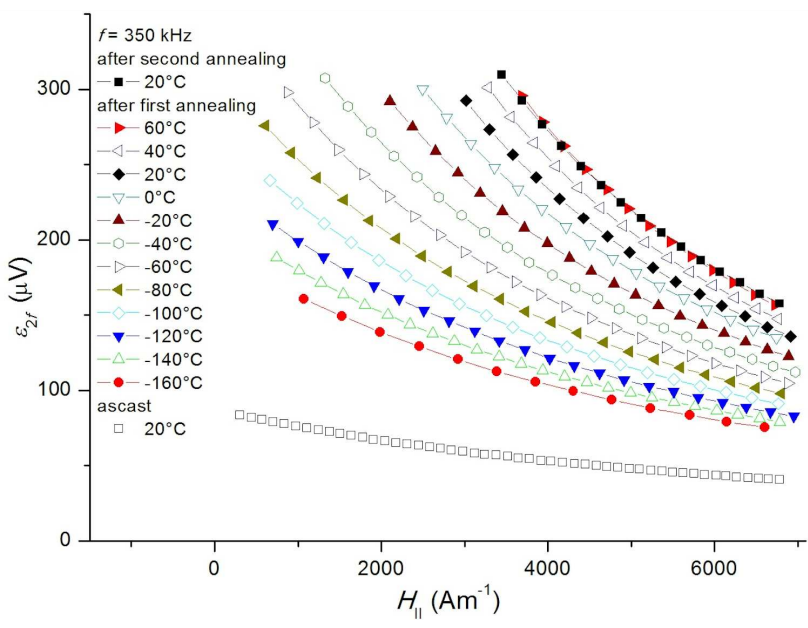

Fig. 3. Influence of annealing and temperature on the induced voltage $\varepsilon_{2 f}$ versus applied axial field dependencies.

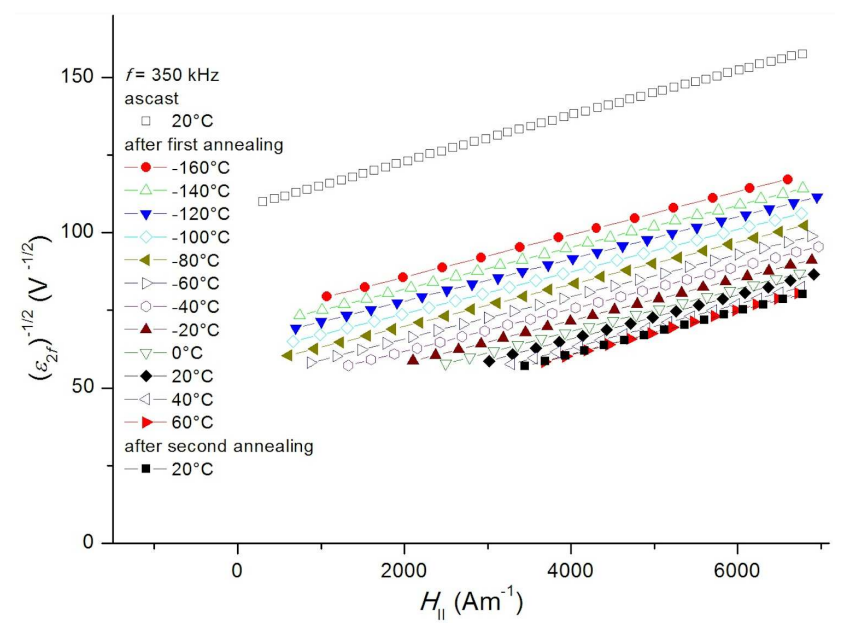

Fig. 4. Influence of annealing and temperature on $1 / \sqrt{\varepsilon_{2 f}}$ versus applied axial field dependencies.

anisotropy field which is produced by interaction between the glass coating and ferromagnetic core of the microwire. Using the value of $H_{\sigma}$, parameters of the microwire $\left(\lambda_{s}=3 \times 10^{-5}\right)$ and the formula for the field of magnetoelastic anisotropy (see comment to (2)), and the axial mechanical stress in the microwire can be calculated. The values are summarized in Fig. 5.

The interaction between the glass coating and ferromagnetic core is mainly determined by the different thermal expansion coefficients of these parts of the microwire. The thermal expansion coefficient of the metal core is larger than that of the glass coating. This means that decreasing temperature results in increasing axial mechanical stress in the ferromagnetic part of the microwire. This effect can be seen in Fig. 5 for the microwire after the first annealing. The annealing combined with slow cooling results in a significant reduction in

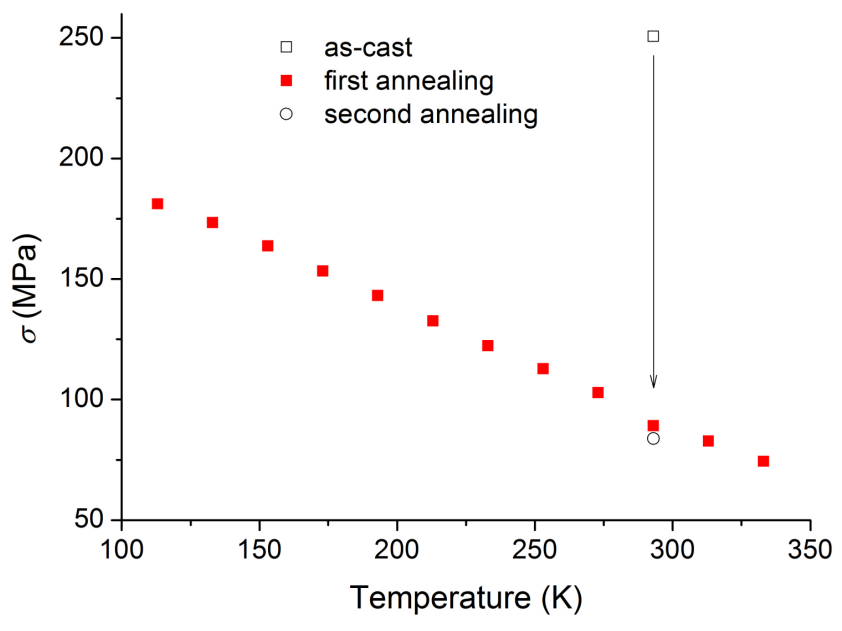

Fig. 5. Axial mechanical stress in the as-quenched microwire and after the first and the second annealings at different temperatures.

mechanical interaction between the two parts of the microwire. The second annealing caused only a slight decrease in the stress. It seems therefore that rapid cooling has no observable effect.

\section{Conclusions}

The specific condition of the SAMR experiment when it is carried out on a single piece of microwire is discussed. The cylindrical shape with small cross-sectional area of the microwire is the reason why some modifications of the standard SAMR experiment are necessary. A transverse AC magnetic field is created by the electric current flowing through the microwire. Due to the low magnitude of the measured signal, AC current of higher frequency (hundreds of $\mathrm{kHz}$ ) has to be used. It is shown that this experiment provides an opportunity to determine the magnetoelastic anisotropy and corresponding axial mechanical stress in the metallic core of the microwire. Interaction between the metallic core and the glass coating is the mechanism predominantly responsible for the observed changes in axial mechanical stress caused by annealing, and also the changes in axial mechanical stress at different temperatures.

\section{Acknowledgments}

This research was supported by VEGA grant No. 1/0388/18 from the Scientific Grant Agency of the Ministry for Education of the Slovak Republic, by the Slovak Research and Development Agency under contract No. APVV-16-0079, and also by the project "Centre of Excellence for Integrated Research \& Exploitation of Advanced Materials and Technologies in Automotive Electronics" ITMS 26220120055. 


\section{References}

[1] J. Onufer, J. Ziman, M. Kladivová, J. Magn. Magn. Mater. 396, 313 (2015).

[2] H. Chiriac, T.A. Ovari, G. Pop, Phys. Rev. B 52 10104 (1995)

[3] J. Onufer, J. Ziman, P. Duranka, M. Kladivova, Phys. B: Cond. Mat. 540, 58 (2018).

[4] R. Varga, Y. Kostyk, K. Richter, A. Zhukov, M. Vázquez, Phys. Status Solidi A 206, 608 (2009).

[5] V. Zhukova, M. Ipatov, A. Zhukov, Sensors 9, 9216 (2009).

[6] C. Morón, C. Cabrera, A. Morón, A. Garcia, M. González, Sensors 15, 28340 (2015).

[7] S.A. Baranov, V.S. Larin, A.V. Torcunov, Crystal 7, 136 (2017).
[8] J. Onufer, J. Ziman, M. Rezničak, S. Kardoš, Acta Phys. Pol. A 131, 723 (2017).

[9] K. Narita, J. Yamasaki, H. Fukunaga, IEEE Trans. Magn. 16, 435 (1980).

[10] O.A. Chubykalo, J. Gonzalez, P. Aragoneses, J.M. Blanco, L. Dominguez, J.M. Gonzalez, J. Magn. Magn. Mater. 169, 169 (1997).

[11] S. Gudoshnikov, M. Churyukanova, S. Kaloshkin, A. Zhukov, V. Zhukova, N.A. Usov, J. Magn. Magn. Mater. 387, 53 (2015).

[12] M. Knobel, M. Vazquez, L. Kraus, in: Giant Magnetoimpedance: Handbook of Magnetic Materials, Vol. 15, Ed. K.H.J Buschow, Elsevier, 2003 p. 497. 